\title{
Prueba de ventilación espontánea en pacientes ventilados. Evaluación del cumplimiento de pautas protocolizadas contra análisis del equipo asistencial
}

Bres. Nicolás Facciola*, Marcela Martínez*, Paula Morgade*, Dr. Arturo Briva ${ }^{\dagger}$

\section{Resumen}

Introducción: la duración ideal de la asistencia ventilatoria mecánica (AVM) genera debate e incertidumbre. El intento de desvinculación prematuro condiciona fracaso y aumento de mortalidad, mientras que la desvinculación tardía aumenta el riesgo del paciente. La utilización de pruebas de ventilación espontánea (PVE) son seguras e identifican aceptablemente a los pacientes listos para desvinculación. Sin embargo, la adherencia a pautas protocolizadas es muy variable y falla la implementación de evidencia científica a nivel clínico.

Objetivo: analizar la interacción entre pautas de desvinculación de AVM y evaluación médica para la toma de decisiones.

Material y método: trabajo descriptivo, prospectivo, con entrevista a médicos en la unidad de cuidados intensivos (UCl) sobre su valoración de la condición del paciente para realizar una PVE. Se comparó la opinión del médico con la evaluación hecha según protocolo de la UCI. Se analizaron coincidencias y discrepancias entre opinión de médicos y protocolo.

Resultados: ingresaron 27 pacientes y 46 médicos. Las coincidencias representaron el 85,4\% de las opiniones aunque existieron elementos de "confusión" en la decisión médica, tanto en coincidencias como en discrepancias. El más frecuente fue el estado de conciencia del paciente.

Discusión y conclusiones: la valoración de la conciencia es fundamental para la asistencia diaria, pero no para la PVE. Su inclusión se vio involucrada en casi un tercio de las respuestas obtenidas, difiriendo la realización de la PVE. Este aspecto debe ser tenido en cuenta tanto para instancias docentes como asistenciales al momento de optimizar los tiempos para PVE y desvinculación de la AVM.

Palabras clave: RESPIRACIÓN ARTIFICIAL

DESCONEXIÓN DEL VENTILADOR

PRUEBAS DE FUNCIÓN RESPIRATORIA

PROTOCOLOS

Key words: ARTIFICIAL RESPIRATION

VENTILATOR WEANING

RESPIRATORY FUNCTION TESTS

PROTOCOLS

\footnotetext{
* Estudiante de Medicina. Hospital de Clínicas. Universidad de la República.

† Prof. Adjunto Cátedra de Medicina Intensiva. Hospital de Clinicas. Universidad de la República.

Correspondencia: Dr. Arturo Briva. Correo electrónico: abriva@hc.edu.uy

Los autores declaran no tener conflicto de interés.

Recibido: $16 / 5 / 17$

Aprobado: $31 / 7 / 17$
} 


\section{Introducción}

La asistencia ventilatoria mecánica (AVM) es una herramienta fundamental en la asistencia de pacientes críticos. Desde sus inicios en el siglo pasado, su utilidad y versatilidad han crecido con la incorporación permanente de tecnología, generando nuevos modos ventilatorios y realizando el soporte vital a diferentes patologías.

Basándonos en estudios reportados y las recomendaciones más recientes, podemos definir algunos puntos importantes en relación con la duración de la AVM, sus efectos secundarios y las estrategias para desvinculación de la misma ${ }^{(1)}$.

Determinar la duración ideal de la AVM siempre será tema de debate e implica cierto grado de incertidumbre. En términos generales se establecen como criterios mínimos que la causa que llevó al paciente a la AVM se encuentre mejorando y que el mismo sea capaz de mantener una ventilación espontánea adecuada.

La identificación de este punto evolutivo es muy importante porque el intento de desvinculación prematuro condiciona el fracaso (con aumento de la mortalidad) ${ }^{(2)}$, y la desvinculación tardía expone al paciente a riesgo innecesario $^{(3)}$. De esta manera, es fundamental contar con protocolos que regulen y homogeinicen el proceso de desvinculación, maximizando los beneficios de la AVM y evitando prolongar la asistencia más tiempo del necesario. De ahí la necesidad de contar con un adecuado ensayo que determine la capacidad de sostener la ventilación espontánea.

En este sentido, múltiples ensayos randomizados han demostrado que la utilización diaria de pruebas de ventilación espontánea (PVE) es segura e identifica aceptablemente a los pacientes listos para la desvinculación, reduciendo además el tiempo de $\mathrm{AVM}^{(4)}$. Por este motivo los protocolos de desvinculación incluyen la realización de PVE de manera programada cuando el paciente cumple con ciertos criterios mínimos, como los incluidos en nuestro protocolo asistencial (figura 1).

Al mismo tiempo que se reconoce la utilidad de aplicar protocolos en UCI, se ha reportado que la adherencia a los mismos oscila entre 10\% y 100\% cuando se compara con percepción guiada por médico a cargo ${ }^{(5-7)}$. Esto constituye, sin dudas, un problema serio para la práctica de la medicina intensiva puesto que introduce una variable dificil de cuantificar al momento de evaluar la efectividad de las estrategias terapéuticas planteadas. No solo debemos tomar en cuenta el éxito o fracaso de las estrategias, sino su nivel de cumplimiento. a

\section{Criterios de inclusión}

Mayor de 18 años

Al menos $24 \mathrm{~h}$ de AVM

Aceptación de consentimiento informado

b

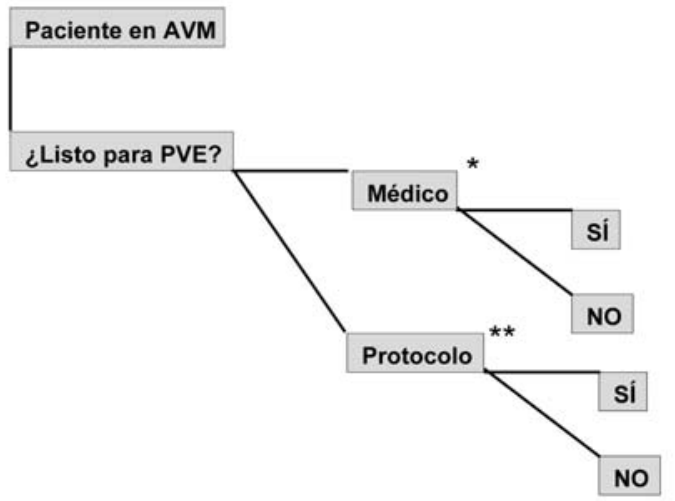

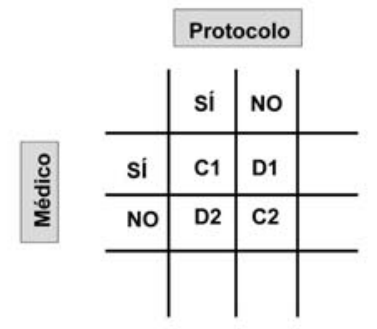

Figura 1. a) Criterios de inclusión para pacientes en estudio; b) Diseño del estudio con consulta a equipo de UCl y evaluación de requisitos del protocolo por historia clínica y monitoreo; c) Diagrama de opiniones y evaluación por protocolo agrupados por coincidencias o discrepancias.

AVM: asistencia ventilatoria mecánica; PVE: prueba de ventilación espontánea; * Entrevista; ** Historia clínica, laboratorio, registro de monitoreo continuo; C1: Grupo de coincidencia 1; C2: Grupo de coincidencia 2; D1: Grupo de discrepancia 1; D2: Grupo de discrepancia 2 
Tomando en consideración todos estos motivos decidimos analizar la interacción entre pautas protocolizadas de desvinculación de la AVM y evaluación de los médicos para la toma de decisiones. Con esta finalidad analizamos la oportunidad de realizar la PVE en pacientes ventilados confrontando la pauta protocolizada con la opinión de los clínicos vinculados a su asistencia. Así esperamos identificar el grado de conocimiento de los criterios pautados, la adherencia de los colegas evaluados hacia el protocolo y la eventual identificación de situaciones que "fuerzan" el abandono del mismo por motivos asistenciales específicos.

\section{Material y método}

El equipo de trabajo se conformó con estudiantes de pregrado cursando el Módulo de Metodología Científica II de la carrera de Doctor en Medicina de la Universidad de la República. El formato del programa es el de tutoría presencial con un docente vinculado al área de trabajo que coordina el proceso de lectura y escritura del proyecto así como la obtención de datos, seguimiento y análisis de los mismos.

Como se observa en la figura 2 (2a y 2 b), los pacientes que cumplieron con los criterios de inclusión fueron valorados diariamente por el equipo investigador y el equipo asistencial. La evaluación por los investigadores consistió en la lectura de las evoluciones diarias de la historia clínica, la paraclínica solicitada y el monitoreo de las últimas 24 horas (evolución de los controles vitales, electrocardiográficos, presión arterial, saturación digital de hemoglobina y nivel de conciencia), sin interactuar con el paciente directamente ni comunicando al equipo asistencial el resultado de su evaluación. Las dudas que surgieran de este análisis se discutieron exclusivamente con el tutor.

Luego de esta instancia, el equipo investigador consultaba la pauta protocolizada para definir si el paciente cumplía con los criterios mínimos de realización de PVE (figura 2$)^{(8)}$.

Posteriormente se encuestó a todos los médicos que en ese día formaban parte del equipo asistencial (asistentes, residentes y posgrados) para saber si el paciente estaba en condiciones o no de realizar la PVE. Si la opinión del médico era que el paciente no podía realizar la PVE, se interrogaba sobre la o las causas que motivaban esa imposibilidad. Debido a que la asistencia es llevada adelante por un equipo, varios médicos podían emitir opiniones sobre un mismo paciente en un solo día o varias veces a lo largo del período de estudio. En cualquier caso, las opiniones fueron obtenidas de manera individual luego de que el médico examinara al paciente y obtuviera toda la información necesaria del monitoreo e

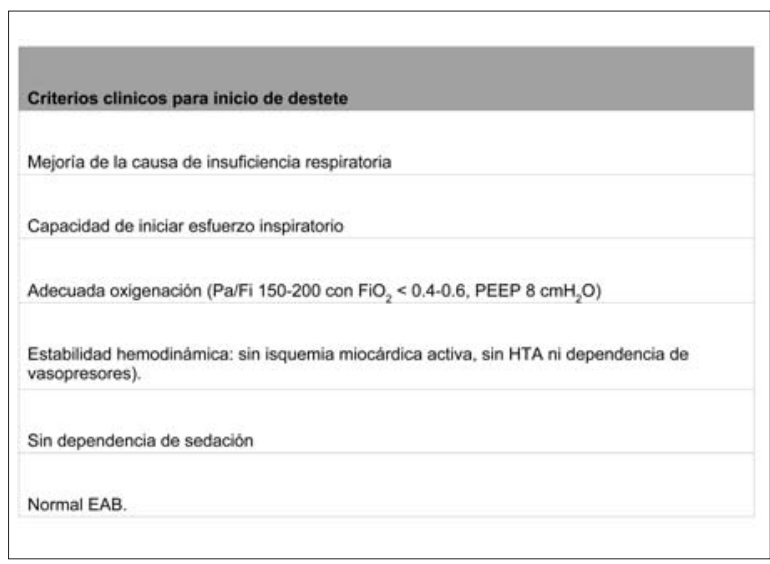

Figura 2. Criterios mínimos para realizar la PVE (tomado de ref 8). EAB: equilibrio ácido base.

historia clínica y sin que los otros integrantes del equipo conocieran su respuesta.

Finalmente se elaboró una planilla de recolección de datos en la que se incluyó la valoración diaria de cada paciente y la respuesta de cada médico a cargo de cada paciente ingresado. De esta manera se establecieron coincidencias o discrepancias entre médico y protocolo.

Este proyecto de investigación fue analizado y aprobado por el Comité de Ética de la Facultad de Medicina y se elaboraron dos formularios de consentimiento informado: uno para familiares de pacientes y otro para los médicos participantes. Al mismo tiempo, el proyecto cumple con los requisitos internacionales de investigación clínica.

Debido al formato del estudio no se compararon poblaciones de pacientes. De esta manera, el análisis de las respuestas se estableció como proporciones del total o de subpoblaciones analizadas.

En un posanálisis se compararon respuestas de médicos, por lo que se utilizó comparación de medias en muestras sin distribución normal (test de D'Agostino-Pearson's para determinar normalidad, ANOVA con test de Friedman para identificar diferencias entre grupos con nivel de significación 0,05 ).

\section{Resultados}

El estudio se desarrolló del 19 de julio al 20 de agosto del 2016, durante 31 días. En ese período se registraron 38 ingresos a UCI, de los cuales 27 ingresaron al estudio. Aceptaron participar 46 médicos, de los que se obtuvieron 339 opiniones.

\section{Análisis global coincidencias/discrepancias}

Luego de obtenidas las respuestas por los médicos interrogados, se compararon las mismas con la valoración del protocolo. 
La figura 3 muestra en un diagrama de doble entrada la presencia de coincidencias (sí/sí o no/no) y discrepancias (sí/no o no/sí) entre médicos y protocolo.

Del total de 339 respuestas, existieron 30 coincidencias sí/sí y 260 coincidencias no/no. De manera global, estas coincidencias representan el $85,4 \%$ de las respuestas, aunque se evaluarán en detalle en otro subanálisis.

Las discrepancias entre médico y protocolo fueron sí/no ( 28 casos, $8,2 \%$ del total) y no/sí ( 21 casos, $6,2 \%$ del total) y serán analizadas también de manera diferencial.

\section{Análisis específico de coincidencias}

De las 290 coincidencias, 260 corresponden a la categoría no/no (figura 2c, grupo C2). Dentro del grupo C2 se observó que en 48 respuestas existió la coincidencia en que el paciente no estaba en condiciones de realizar la PVE, pero los motivos expuestos por los médicos no estaban incluidos en el protocolo. El motivo más frecuentemente identificado en esta categoría fue el "nivel de conciencia no adecuado" (27 casos, 56,2\%).

De las restantes 212 respuestas del grupo C2, en 184 casos existió una coincidencia total entre el motivo identificado por el médico y el requisito del protocolo para PVE $(54,2 \%)$. En los 28 casos restantes existió coincidencia, pero además el médico incluyó motivos para su opinión no establecidos en el protocolo. En este último subgrupo el motivo más frecuente también fue el "nivel de conciencia no adecuado" (14 casos, 50\%). La figura 2 resume la distribución de todos los subgrupos de análisis.

\section{Análisis específico de discrepancias}

Cuando la discrepancia fue sí/no (figura $2 \mathrm{c}$, grupo D1), el principal ítem del protocolo que recomendaba no realizar la PVE, y que no fue identificado por los médicos, fue la incapacidad para iniciar el esfuerzo inspiratorio (13 de 28 casos, 46,4\%), seguido por ausencia de mejora en causa de insuficiencia respiratoria (8 casos, $28,5 \%$ ) e inadecuada oxigenación (5 casos, $17,8 \%)$.

En el sentido opuesto, cuando el médico consideraba que el paciente no podía realizar la PVE, aunque el protocolo lo avalara (discrepancia no/sí, grupo D2) los motivos de esta discrepancia fueron variados. Ordenados por frecuencia descendente las respuestas fueron: GCS bajo (5/21), no tolera tubo T (4/21), sospecha (no documentada) de inicio de actividad infecciosa $(4 / 21)$, traslado a block quirúrgico en las siguientes 24 horas $(2 / 21)$, adecuación del esfuerzo terapéutico $(2 / 21)$, mal manejo de secreciones $(1 / 21)$.

\section{Identificación de condiciones e inicio de PVE}

En 51 de las 339 opiniones recogidas el protocolo avalaba la realización de la PVE, independientemente de la opinión (coincidente o no) del médico encuestado.

En este subgrupo se analizó cuánto tiempo transcurría desde que el paciente cumple los requisitos del protocolo hasta que efectivamente se realiza la PVE.

Las 51 opiniones correspondieron a 12 pacientes (44,4\% de los ingresados al estudio). En estos pacientes la PVE se realizó 1,5 días después que el protocolo ya respaldaba su realización (mediana 1,5 días máx $=2,3$ mín =0,6).

Utilizando la historia clínica se examinaron los motivos registrados en ella que pudieran explicar la demora. Dentro de los mismos se destacan: "necesidad de revalorar respuesta neurológica" (45\%), "hipodinamia" (26\%), "realización de traslados o instrumentación del paciente" (10\%) y "sin causa descrita" $(10 \%)$.

\section{Rol del nivel de conciencia en la toma de decisiones}

Al observar la presencia de la valoración del nivel de conciencia, asociado o no a la GCS en casi todas las situaciones exploradas, decidimos realizar un postest para avanzar en su análisis. Se elaboró un cociente entre opiniones que incluían el GCS y las opiniones totales aportadas (figura 4). Al mismo tiempo se subdividieron las respuestas entre aquellas obtenidas de médicos en formación (posgrados y residentes) y médicos de guardia intensivistas recibidos (asistentes).

Se observaron los cocientes para cada colega participante y se estableció la media y el error estándar para la población total $(\mathrm{n}=46 ; 0,267 \pm 0,03)$, el subgrupo de asistentes $(\mathrm{n}=13 ; 0,261 \pm 0,04)$ y para médicos en formación como intensivistas $(\mathrm{n}=33 ; 0,269 \pm 0,04)$. No se encontraron diferencias significativas entre los grupos $(\mathrm{p}=0,4551)$.

\section{Discusión}

La ventilación mecánica ha sido parte fundamental del soporte vital a pacientes críticos desde sus inicios en la epidemia de polio en $1952^{(9)}$. Casi al mismo tiempo se observó la necesidad de conocer los mecanismos que permiten desvincular al paciente que recibe el soporte ventilatorio de manera exitosa ${ }^{(10)}$. La desvinculación de la AVM hace referencia al proceso por el cual los pacientes se liberan de la dependencia del soporte mecánico, permitiendo su ventilación espontánea sin la necesidad de acceso invasivo o no invasivo a la vía aérea.

De esta manera, a lo largo de las últimas décadas ha sido exponencial la publicación de reportes relacionados a este tema. Esto incluye estrategias específicas, nuevos modos ventilatorios para lograr la desvincula- 


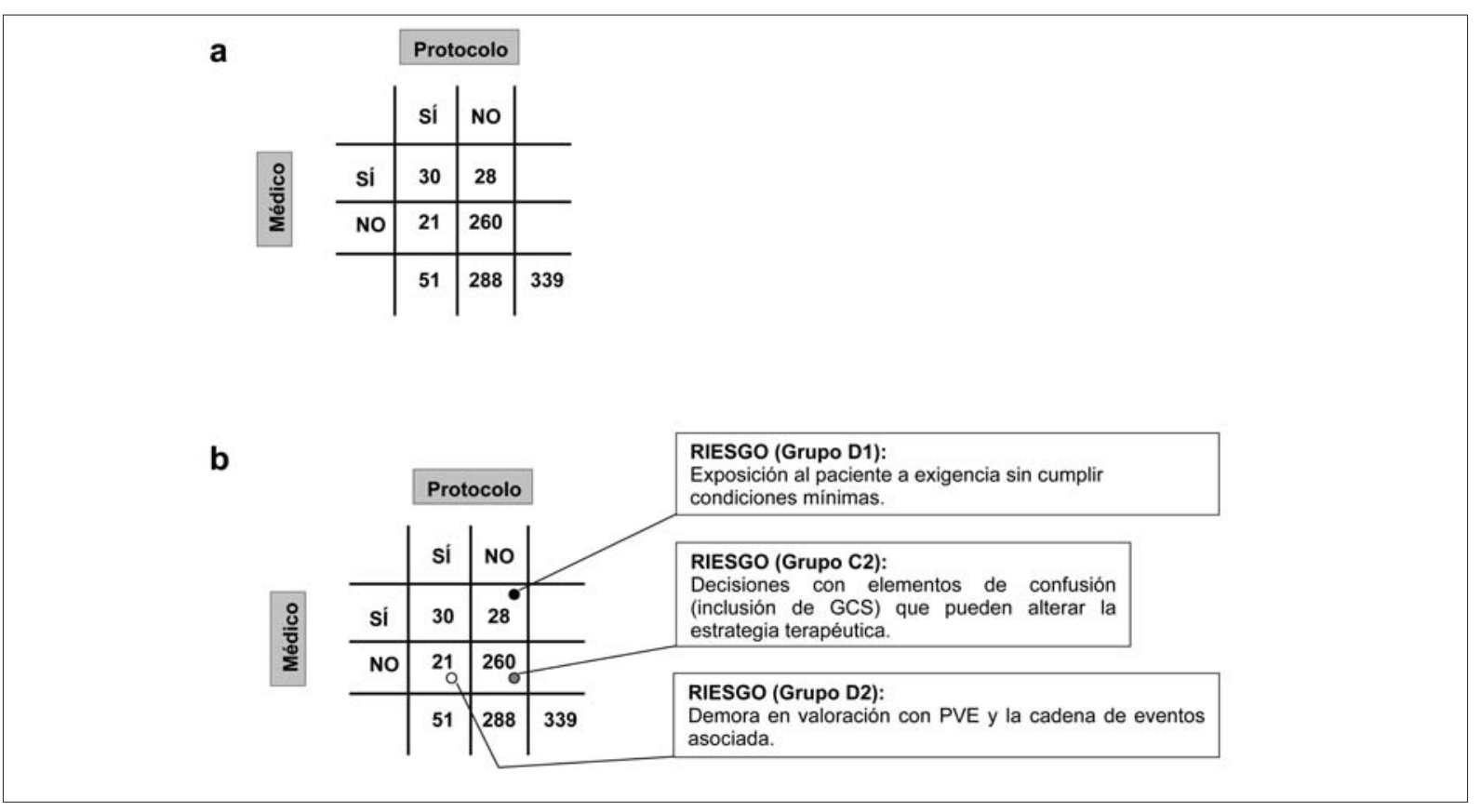

Figura 3. Esquema de distribución opiniones/evaluación agrupadas por coincidencias o discrepancias. Cada casilla representa la cantidad de eventos registrados (a) junto con los potenciales riesgos asociados a la toma de decisiones basadas en el resultado obtenido (b).

ción y la elaboración de predictores de éxito o fracaso del proceso ${ }^{(1)}$.

Sin embargo, uno de los pasos limitantes para completar adecuadamente el proceso de desvinculación es la identificación de los pacientes que están listos para realizarla. De hecho, cerca del $40 \%$ del tiempo de soporte ventilatorio se utiliza para la desvinculación del pacien$\mathrm{te}^{(11)}$ y la prolongación innecesaria de la AVM aumenta la mortalidad, la neumonía asociada a la ventilación, la lesión pulmonar y la estadía hospitalaria ${ }^{(2)}$.

Al mismo tiempo, la realización de una PVE protocolizada, junto a la valoración clínica diaria, aumentan las chances de extubación exitosa y disminuye la duración de la AVM en adultos ${ }^{(6)}$, por lo que es una estrategia ampliamente recomendada ${ }^{(4)}$.

Esta aproximación al paciente ventilado implica la toma de decisiones basada en un conjunto de elementos cuantificables junto a percepciones individuales incluidas dentro del concepto de valoración clínica. Esto constituye un desafío tanto para el diseño de un protocolo de desvinculación como para evaluar su implementación y eficacia, puesto que la decisión final no se resume en una operación aritmética sino en una conjunción de evaluación y percepción.

Por este motivo desarrollamos un diseño experimental que se enfoca en valorar algunos aspectos del uso y conocimiento del protocolo, identificando además qué otros elementos pesan en la percepción del médico al momento de valorar la oportunidad de realizar una PVE en el paciente ventilado en UCI.

En nuestro ensayo hemos podido cuantificar algunas situaciones que reflejan esta combinación de factores. Se observó que existe un nivel basal de coincidencias médico-protocolo alto (184 casos de $339 ; 54,2 \%$ ) y un bajo nivel de desconocimiento del protocolo como principal causa de discrepancia (27 casos de 339; 7,96\%).

Sin embargo, existe una serie de datos "de confusión" en la toma de decisiones que deben evaluarse detenidamente porque influyen claramente en la percepción de los médicos.

En el diagrama de la figura $3 \mathrm{~b}$ volvemos a presentar los resultados obtenidos del ensayo, pero ahora con lo que llamamos "áreas de riesgo", o sea, cómo interpretamos que influirían esas respuestas en la toma de decisiones y qué riesgo correrían los pacientes en cada una de ellas.

Cuando la evaluación del colega avala la PVE, pero el protocolo no lo respalda (grupo D1), el paciente es sometido a una exigencia para la que eventualmente no está preparado. Si bien el protocolo no es inalterable, debe reevaluarse qué condición no cumple el paciente y decidir si es seguro avanzar en PVE a pesar de no estar recomendado. Desde el punto de vista operativo esto implica reforzar el conocimiento de las pautas protocolizadas para evitar las decisiones inadecuadas o establecer el riesgo clínico de realizar la PVE sin seguir la pauta. 
En el segundo grupo de discrepancia (evaluación del médico es no y el protocolo avala realizar PVE, grupo D2), el mayor riesgo identificado es la demora en realizar la PVE. En nuestro trabajo se observó una demora de 1,5 días en realizar la prueba, lo que conlleva un eventual retraso en las decisiones posteriores (permanencia en AVM la más importante). Nuestra UCI recibe habitualmente pacientes neurocríticos en los que la valoración del estado de conciencia a través del GCS es parte fundamental de la asistencia, aunque no es así para la realización de la PVE. En el paciente con lesión cerebral la demora en la extubación, cuando cumple con criterios para la misma, agrega morbilidad, mayor riesgo de neumonía, mayor estadía en UCI y hospitalaria ${ }^{(12)}$, por lo que este es un aspecto a corregir en el futuro.

En el tercer grupo, a pesar de tratarse de coincidencias entre evaluación médica y protocolo (ambos rechazan realizar PVE, Grupo C2) existen elementos de confusión para la toma de decisiones. El principal elemento identificado fue nuevamente la inclusión del nivel de conciencia del paciente. Si bien al coincidir en la negativa a realizar la PVE efectivamente el GCS no influyó en la decisión clínica, es probable que pueda condicionar futuras decisiones si en la evolución el paciente alcanza los requerimientos mínimos del protocolo pero persiste con nivel de conciencia alterado. A lo expresado sobre el Grupo D2 se suma que varios estudios demostraron que el GCS no es predictor de fracaso en desvinculación ni de neumonía por aspiración, a pesar de que es uno de los motivos mencionados por los médicos como negati$\mathrm{vo}^{(13)}$. Al mismo tiempo, la proporción de las opiniones que tomaba en cuenta el GCS dentro de las respuestas obtenidas fue cercana a $29 \%$, sin diferencias significativas entre los dos niveles de formación (docentes con título de intensivistas y médicos con formación parcial en medicina intensiva). Esto nos induce a pensar que la inclusión de este factor de confusión no depende de la experiencia o "visión clínica" del médico interrogado (figura 4). Corresponde probablemente a un sesgo inducido por el objetivo final de desvincular al paciente de la AVM.

Evidentemente el objetivo último sigue siendo la desvinculación total del paciente del equipo de AVM, pero esto no debería confundirse con el rol de la PVE en el proceso de desvinculación. La PVE permite determinar, cuando un grupo de condiciones mínimas se encuentran presentes, si existen dificultades para una eventual desconexión y no un elemento pronóstico de la misma. Por este motivo, si el paciente supera la PVE podremos considerarlo candidato a seguir avanzando en la desvinculación; pero esto no implica que la desconexión sea mandatoria ni mucho menos exitosa.

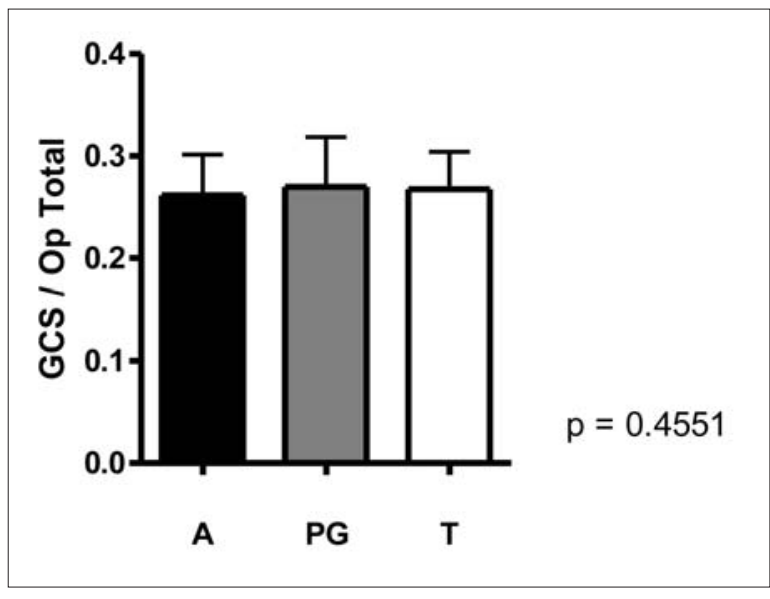

Figura 4. Proporción de opiniones que involucraban el nivel de conciencia del paciente en función de las respuestas totales obtenidas de los médicos entrevistados. GCS: Glasgow Coma Scale; A: asistentes (intensivistas docentes); PG: posgrados de medicina intensiva; T: totalidad de respuestas obtenidas. P: valoración de significación estadística entre grupos estudiados para test ANOVA con postest de Friedman.

En este contexto impresiona que los médicos evaluados tienden a preseleccionar a los pacientes que llegan a la PVE, realizándola solo a quienes creen que podrán superarla. Esto implica que se realiza una selección de riesgo en un punto incorrecto del algoritmo de desvinculación y que puede inducir a decisiones clínicas incorrectas. Existen múltiples predictores de riesgo para el fracaso de la desvinculación, pero no realizar la PVE en pacientes que cumplen con los criterios para ella nos impide obtener valiosa información, fundamentalmente en los que no logran completarla.

La figura 5 plantea un esquema de manejo a propósito de este punto que podría incluirse en cualquier protocolo de desvinculación de la AVM.

Como parte del análisis global hemos identificado algunas debilidades en este proyecto que merecen ser discutidas. Los patrones de confusión que determinan modificaciones de la evaluación médica no necesariamente reflejan modificaciones en la toma de decisiones porque los entrevistados tienen diferente peso en las opciones terapéuticas elegidas. Sin embargo, a pesar de que nuestra evaluación incluye la opinión de colegas en formación y que no necesariamente toman las decisiones asistenciales, la incidencia del principal factor de confusión (GCS) fue similar en los dos grupos de médicos. En cualquier caso, la identificación de este patrón de percepción debe ser tenido en cuenta para reforzar tanto las instancias docentes como de discusión clínica en el futuro. 


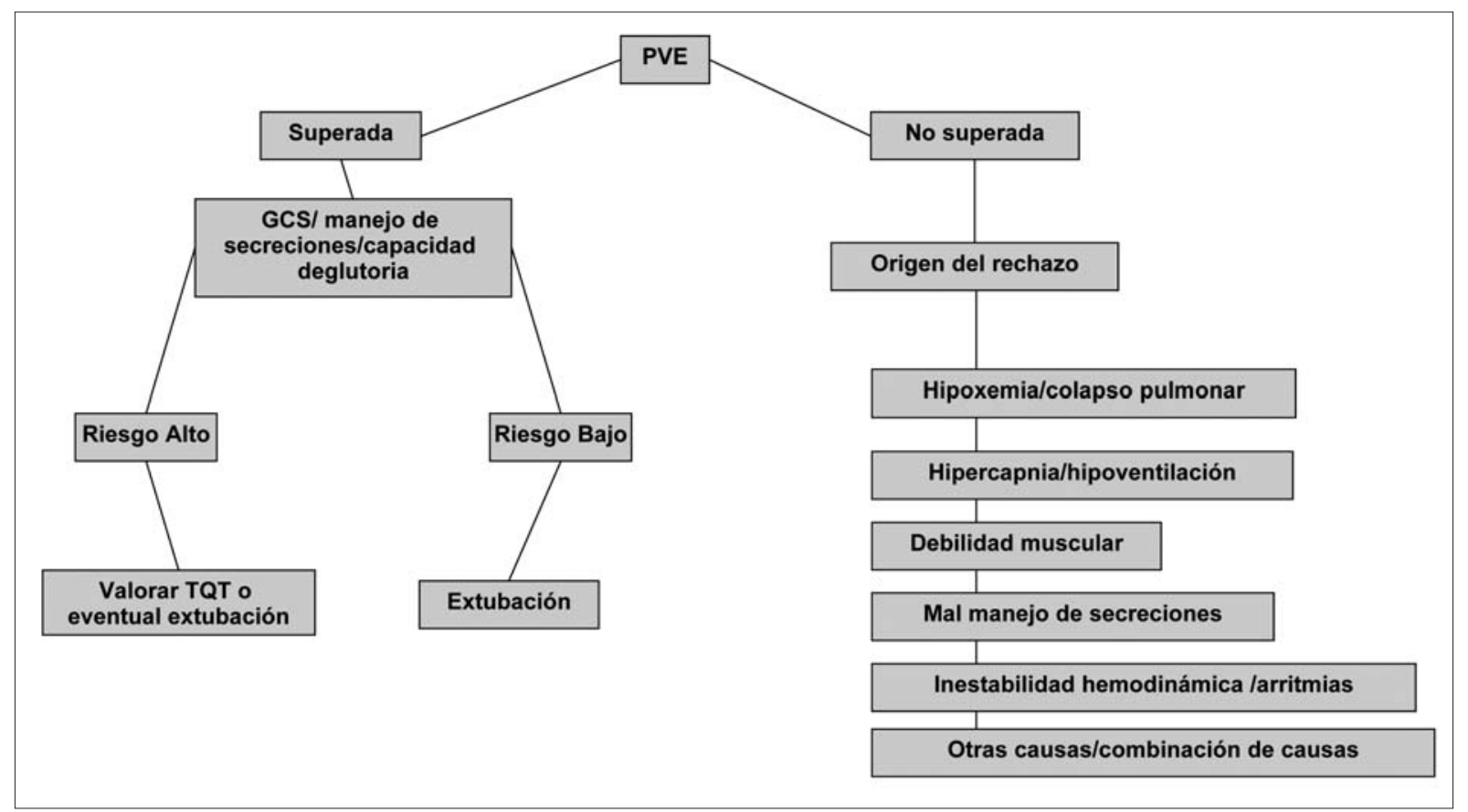

Figura 5. Esquema básico para insertar la PVE en cualquier protocolo general de desvinculación de la AVM, incluyendo el nivel de conciencia de los pacientes.

Por otro lado, la evaluación de la capacidad de iniciar el ciclo respiratorio puede estar relacionada con el grado de adaptación a la AVM o el intercambio gaseoso del paciente, por lo que los diferentes niveles de entrenamiento podrían condicionar la respuesta obtenida. Lamentablemente el diseño del estudio no incluyó la valoración del desempeño directo del médico con el paciente y su monitoreo, por lo que no pudo explorarse un perfil diferente en función del entrenamiento.

En términos generales consideramos que los resultados obtenidos respaldan nuestras presunciones iniciales sobre las dificultades de aplicación de cualquier protocolización clínica ${ }^{(14)}$. De hecho, la falla en la implementación de evidencia científica dentro de la práctica clínica es uno de los desafíos más grandes de la medicina actual y su corrección debe ser una tarea de construcción permanente en UCI.

Esto no implica desconocer que la práctica de la medicina, y especialmente de la medicina intensiva, obliga a que en tiempos reducidos el médico debe tomar decisiones gestionando la incertidumbre (tanto por falta de información como por exceso de datos que deben ser priorizados) y que esto no siempre es reflejado por un protocolo. Sin embargo, creemos necesario realizar este tipo de experiencias sistematizadas y cuantificables para reconocer fortalezas y debilidades de las pautas protocolizadas y su aplicación a la práctica clínica diaria.

\section{Abstract}

Introduction: Ideal duration of mechanical ventilation is a source of debate and uncertainty. Early weaning attempts result in failure and increased mortality rates, while a late discontinuation of ventilation increases the patients' risks. The use of the spontaneous ventilation test is safe and results in a fair identification of patients who are ready for weaning. However, adherence to protocol guidelines varies and scientific evidence fails to be implemented in the clinical practice.

Objective: to analyze the interaction between mechanical ventilation discontinuation guidelines and medical assessment for the making of a decision.

Method: descriptive, prospective study which involved interviewing physicians in the Intensive Care Unit on their assessment of the patient's condition to perform a spontaneous ventilation test. The physicians' opinion was compared to the assessment carried out as per the Intensive Care Unit Protocol, in order to analyze agreements and discrepancies.

Results: 27 patients and 46 physicians were included in the study. Agreements represented $85.4 \%$ of opinions, although there were a few confusing elements confusing as to the medical decision to be made, both in terms of agreements and discrepancies, the most frequent of which was the patient's level of consciousness.

Discussion and conclusions: assessing the level of consciousness of patients is essential in the daily practi- 
ce of medicine, although not for the spontaneous ventilation test. It was included in almost one third of the responses obtained and delayed the performance of a spontaneous ventilation test. This fact needs to be considered both in the context of training instances and at the time of optimizing times for the spontaneous ventilation test and the discontinuation of mechanical ventilation.

\section{Resumo}

Introdução: a duração ideal da Assistência Ventilatória Mecânica (AVM) gera debate e incerteza. A tentativa de desmame precoce leva ao fracasso e ao aumento da mortalidade, enquanto o desmame tardio aumenta o risco do paciente. A utilização de testes de ventilação espontânea (TVE) são seguras e identificam razoavelmente os pacientes que já estão em condições para o desmame. No entanto, a adesão a pautas protocolizadas é muito variável e a implementação de evidencia científica no nível clínico falha.

Objetivo: analisar a interação entre as pautas de desmame de AVM e a avaliação médica para a tomada de decisões.

Material e método: trabalho descritivo, prospectivo, com entrevista a médicos na Unidade de Cuidados Intensivos (UCI) sobre sua avaliação da condição do paciente para realizar um TVE. A opinião do médico e a avaliação feita de acordo com o protocolo da UCI foram comparadas. As coincidências e discrepâncias entre opinião de médicos e protocolo foram analisadas.

Resultados: 27 pacientes e 46 médicos foram incluídos. As coincidências representaram 85,4\%, das opiniões mesmo quando se observaram elementos de "confusão" na decisão médica, tanto em coincidências como discrepâncias. O mais frequente estava relacionado com o estado de consciência do paciente.

Discussão e conclusões: a avaliação da consciência é fundamental para a assistência diária, mas não para o TVE. Sua inclusão estava presente em quase um terço das respostas obtidas, com diferencias sobre a realização do TVE. Este aspecto deve ser considerado tanto na docência como na assistência para otimizar os tempos para a realização de TVE e do desmame da AVM.

\section{Bibliografía}

1. McConville JF, Kress JP. Weaning patients from the ventilator. N Engl J Med 2012; 367(23):2233-9.

2. Frutos-Vivar F, Esteban A, Apezteguia C, González M, Arabi Y, Restrepo MI, et al. Outcome of reintubated pa- tients after scheduled extubation. J Crit Care 2011; 26(5):502-9.

3. Thille AW, Cortés-Puch I, Esteban A. Weaning from the ventilator and extubation in ICU. Curr Opin Crit Care 2013; 19(1):57-64

4. Fan E, Zakhary B, Amaral A, McCannon J, Girard TD, Morris PE, et al. Liberation from mechanical ventilation in critically ill adults: an official ATS/ACCP clinical practice guideline. Ann Am Thorac Soc 2017; 14(3):441-443.

5. Burns SM, Earven S, Fisher C, Lewis R, Merrell P, Schubart JR, et al; University of Virginia Long Term Mechanical Ventilation Team. Implementation of an institutional program to improve clinical and financial outcomes of mechanically ventilated patients: one-year outcomes and lessons learned. Crit Care Med 2003; 31(12):2752-63.

6. Duane TM, Riblet JL, Golay D, Cole FJ Jr, Weireter LJ Jr, Britt LD. Protocol-driven ventilator management in a trauma intensive care unit population. Arch Surg 2002; 137(11):1223-7.

7. Krishnan JA, Moore D, Robeson C, Rand CS, Fessler HE. A prospective, controlled trial of a protocol-based strategy to discontinue mechanical ventilation. Am J Respir Crit Care Med 2004; 169(6):673-8.

8. Santos C, Alzugaray P. Desvinculación de la ventilación mecánica. En: Biestro A, ed. CTI protocolos. Montevideo: Cuadrado, 2015:273-80.

9. Lassen HC. A preliminary report on the 1952 epidemic of poliomyelitis in Copenhagen with special reference to the treatment of acute respiratory insufficiency. Lancet 1953; 1(6749):37-41.

10. Bushnell LS, Pontoppidan H, Hedley-Whyte J, Bendixen HH. Efficiency of different types of ventilation in long-term respiratory care: mechanical versus spontaneous. Anesth Analg 1966; 45(5):696-703.

11. Peñuelas Ó, Thille AW, Esteban A. Discontinuation of ventilatory support: new solutions to old dilemmas. Curr Opin Crit Care 2015; 21(1):74-81.

12. Godet T, Chabanne R, Marin J, Kauffmann S, Futier E, Pereira B, et al. Extubation failure in brain-injured patients: risk factors and development of a prediction score in a preliminary prospective cohort study. Anesthesiology 2017; 126(1):104-114.

13. Coplin WM, Pierson DJ, Cooley KD, Newell DW, Rubenfeld GD. Implications of extubation delay in brain-injured patients meeting standard weaning criteria. Am J Respir Crit Care Med 2000; 161(5):1530-6.

14. Briva A, Gaiero C. Lung protection: an intervention for tidal volume reduction in a teaching intensive care unit. Rev Bras Ter Intensiva 2016; 28(4):373-9. doi:10.5935/0103-507X.20160067. 